OPEN ACCESS

Edited by:

Ami Rokach,

The Center for Academic Studies,

Israel

Reviewed by:

Weili Lu,

Rutgers, The State University of New Jersey, United States

Vrinda Kalia,

Miami University, United States

*Correspondence:

Danping Liu

liudanping03@163.com

Specialty section:

This article was submitted to

Personality and Social Psychology,

a section of the journal

Frontiers in Psychology

Received: 22 May 2020

Accepted: 13 August 2021

Published: 08 September 2021

Citation:

Liu Y, Meng H, Conner KO,

Qiao M and Liu D (2021) The

Influence of Health Literacy and Social

Support on Loneliness Among Patients With Severe Mental Illness

in Rural Southwest China.

Front. Psychol. 12:564666.

doi: 10.3389/fpsyg.2021.564666

\section{The Influence of Health Literacy and Social Support on Loneliness Among Patients With Severe Mental Illness in Rural Southwest China}

\author{
Yan Liu',2, Hongdao Meng ${ }^{3}$, Kyaien O Conner ${ }^{4}$, Mutian Qiao ${ }^{1}$ and Danping Liu ${ }^{1 *}$ \\ ${ }^{1}$ Department of Health Behavioral and Social Medicine, West China School of Public Health and West China Fourth \\ Hospital, Sichuan University, Chengdu, China, ${ }^{2}$ Department of Academic Affairs, West China School of Medicine/West \\ China Hospital, Sichuan University, Chengdu, China, ${ }^{3}$ School of Aging Studies, College of Behavioral and Community \\ Sciences, University of South Florida, Tampa, FL, United States, ${ }^{4}$ Department of Mental Health Law \& Policy, College \\ of Behavioral \& Community Sciences, University of South Florida, Tampa, FL, United States
}

Loneliness is an important risk factor for poor health outcomes among adults, especially among those with severe mental illnesses (SMls). Existing research has shown that adults with SMls often lack health literacy, which contributes to more restricted social networks and low levels of social support. The objective of this cross-sectional study was to examine the influence of health literacy and social support on the loneliness of patients with SMI in rural Southwest China. We recruited 300 patients with SMI in rural Southwest China between December, 2017 to May, 2018 via a multi-stage stratified random sampling approach. We used structural equation modeling (SEM) test the hypothesized relationships among the variables of the 270 patients who completed the survey. Results of the SEM showed that health literacy was both directly and indirectly associated with loneliness, with social support playing a mediating role. These findings suggest psychoeducation for SMI patients, and their informal caregivers, may offer beneficial effects toward reducing loneliness in this vulnerable population. Further, social support is another potential target for intervention development for improving patient outcomes.

Keywords: Ioneliness, health literacy, social support, severe mental illness, rural, China

\section{INTRODUCTION}

Patients with severe mental illness (SMI) are often described as a group of heterogeneous people who suffer from severe psychiatric disorders together with long-term mental disturbances such as schizophrenia, bipolar disorder, schizoaffective disorders, major recurrent depressive disorder and personality disorders (Jansen, 2018). Patients with SMI often require high levels of care and consume many social and health resources available within the psychiatric and social healthcare network (Nguyen et al., 2020). SMIs contribute significantly to the global burden of disease, impacting more than $4 \%$ of the adult population worldwide and leading to substantial premature deaths, as patients with SMI die on average one to two decades earlier than the general population 
(Wong et al., 2020). In China, with a population of 1.4 billion, the lifetime prevalence of SMI was as high as $10.10 \%$ (Wang et al., 2017). SMIs are disabling, lethal and often require long-term, complex interventions for both SMI patients and their family caregivers (Vigo et al., 2016).

SMI patients may have fewer successful interactions or have limited social networks because deficiencies in certain social and personal functioning skills such as memory deficits and social anhedonia (Barkus and Badcock, 2019). And they are often excluded socially because of societal stigma against individuals with psychiatric symptoms such as depression or paranoia (Schwartz and Gronemann, 2009). In some cases, individuals with SMI might internalize stigma of mental illness. To be specific, they may feel that having psychopathology makes them perform oddly in social situations or that such performance will be embarrassing, then perhaps they they will create negative affect like feeling inferior and steering clear of people because they have adopted beliefs in the larger society that SMI patients are subordinate to everyone else (Prince et al., 2018). All these experience can increasing the likelihood of feeling lonely. Loneliness, has generally been defined as an unpleasant, distressing, and aversive experience or feeling resulting from the discrepancy between one's actual versus expected interpersonal relationships (Tharayil, 2007). People who suffer from two or three mental disorders were shown to have approximately 21 -fold increase in the odds of loneliness, compared to those with no mental disorder (Meltzer et al., 2013). In addition, it has been reported that loneliness might be part of the causal pathway in the development of psychotic experiences (van der Werf et al., 2010). Loneliness has also been associated with a variety of adverse outcomes including increased risk of relapse, psychiatric hospitalization, medical sequelae, and further social disengagement (GayerAnderson and Morgan, 2013; Prince et al., 2018). Studies have found that patients' subjective recovery from psychosis was significantly related to a decrease in loneliness (Roe et al., 2011), and that of adequate health literacy, social support, and social networks may reduce loneliness among SMI patients (Perese and Wolf, 2005).

Health literacy is commonly defined as having the cognitive ability, knowledge and social skills to obtain, process, and use basic health information and services needed to maintain health and make health care decisions (Medicine, 2004). In China, the correct response rate on the Mental Health Knowledge Questionnaire of the general public was $77.0 \%$ (Wang et al., 2013). SMI patients generally report low levels of health literacy (Clausen et al., 2016). Low health literacy is associated with being lowly educated, living in suburban sites and cognitive impairment (Yost et al., 2013). Studies of Masi and colleagues suggest that loneliness can be reduced by addressing abnormal cognition and social skills training (Masi et al., 2011). Geboers et al. (2016) found an association between the lack of health literacy and an increase of loneliness among older adults (Geboers et al., 2016). Previous studies also suggest that poor health literacy in older adults was related to their impoverished social networks and may lead to the loss of necessary social support (Yang et al., 2019).
The notion that sufficient social support might buffer and alleviate the adverse health consequences of low health literacy among different groups [like smokers (Stewart et al., 2014) and patients with chronic kidney disease (Lora et al., 2011)] has also been tested.

Social support is a multidimensional concept generally referering to the social resources or help that persons perceived and received (Kang et al., 2018), which can be reflected in three aspects: the perception of support, the provision of support and the structure of social networks (Gayer-Anderson and Morgan, 2013). Lack of social support has been shown to be a strong predictor of loneliness in persons with psychotic disorders (Schwartz and Gronemann, 2009; Chrostek et al., 2016). Perese and collegaues (Perese and Wolf, 2005) found that patients with SMI reportedly experience higher levels of loneliness than the general population, which is linked to low levels of social support. It is well-known that social support has been frequently considered an important resource and is an important component of interventions targeted at reducing loneliness (Hawkley and Cacioppo, 2010; Masi et al., 2011). Previous findings have revealed the improvements in older adults' social support is due to participation in an exercise intervention which, directly predicted reductions in loneliness (McAuley et al., 2000). Social support may mediate the relationship between other factors (like emotional intelligence) and loneliness (Zou, 2014).

Loneliness, also referred to as "subjective sense of social isolation," may have a salient impact on the emotional wellbeing, social interaction and recovery process among SMI patients (Linz and Sturm, 2013). Given that loneliness in one person can influence loneliness levels in individuals they interact with (Cacioppo et al., 2009), and family members of SMI patients usually experience substantial caregiver burden (Yu et al., 2017), examining strategies to alleviate loneliness in this disadvantaged population who often feel stigmatized, shamed, isolated and who often report lower health literacy and impoverished social support remains important for clinicians, researchers and policymakers. To date, most previous researches have been conducted to investigate factors influencing loneliness (e.g., health literacy and social support) among different populations. However, the importance of health literacy in loneliness is still rarely assessed, and even less evidence is available about the interrelationships and potential mechanisms of health literacy, social support and loneliness among SMI patients.

\section{CURRENT STUDY}

The objective of this study was to examine the influence of health literacy and social support on the loneliness of patients with SMI in rural Southwest China. Based on the results of previous studies, we developed a single confirmatory mediator model shown in Figure 1. Specifically, health literacy had a direct positive correlation with social support (hypothesis 1) and had a direct negative correlation with loneliness (hypothesis 2); social support had a direct negative 


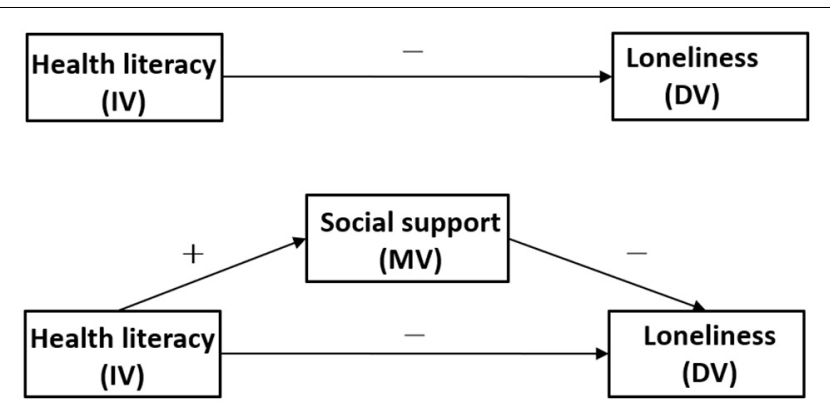

FIGURE 1 | The theoretical model and hypotheses.

correlation with loneliness (hypothesis 3). In addition, we also hypothesized that the relationship between health literacy and loneliness was mediated by social support (hypothesis 4). This study is the first to explore the influence of health literacy and social support on loneliness among patients with SMI in rural China.

\section{MATERIALS AND METHODS}

\section{Participants and Procedure}

This cross-sectional research was conducted among SMI patients in Sichuan province, Southwest China from December, 2017 to May, 2018. The target population was the permanent residents aged 18 years or older who had been diagnosed with SMI.

A multi-stage stratified random sampling approach was used to acquire the sample (Pan et al., 2018). In the first stage, we randomly chose a city in Sichuan province. In the second stage, we randomly selected a rural district in the city. In the third stage, ten townships were randomly selected from the rural district. In the fourth stage, we randomly selected 30 SMI patients from the database of SMI patients established by each township hospital. Township hospitals are required to receive patients with severe mental illness clearly diagnosed by mental health institutions among permanent residents within their jurisdiction, input patients' information into the database, and provide intervention, follow-up and physical examination services for them. Acceptance into services at township hospitals required meeting relevant "diagnostic criteria" in the "Classification of Mental and Behavioral Disorders" section (ICD-10, F00-F99) of the International Classification of Diseases (WHO, 1992) for severe mental illness, which included but is not limited to a diagnosis of Schizophrenia; schizoaffective disorder, paranoid psychosis, bipolar disorder, epilepsy with psychotic features (Hyman et al., 2011). Based on census information from township hospitals, a close approximation of the diagnositc breakdown of the participants in this study as follows: collectively $83.0 \%$ were diagnosed with schizophrenia, $14.4 \%$ with mental retardation with psychotic features, $1.9 \%$ with epilepsy with psychotic features, and $0.7 \%$ with other psychiatric disorders (bipolar disorder, major recurrent depressive), 30.4\% was taking antipsychotic medications. Patients were excluded if deemed by their clinician to have insufficient capacity to consent and respond at the time of assessment. A total of 300 eligible SMI patients were interviewed face-to-face by professionally trained investigators and 270 SMI patients completed the questionnaire (for a valid response rate of $90.0 \%$ ).

\section{Ethical Considerations}

The study protocol was approved by the Institutional Review Board of Sichuan University (Project identification code: H171260). Informed consent was obtained from each SMI patients following a detailed explanation about the purpose of this research.

\section{Measures}

SMI patients' socio-demographics, health literacy, social support and loneliness information were collected from questionnaires.

\section{Socio-Demographics}

Socio-demographics included gender, age, marital status, education level and per capita annual income of the household.

\section{Health Literacy}

Health literacy was assessed by the subscale from the Questionnaire of Mental Health Work issued by the Chinese Ministry of Health: the Mental Health Knowledge Questionnaire (MHKQ) (Liang et al., 2011; Wang et al., 2013). The questionnaire used in this study included 16 items, such as "Mental disorders are caused by incorrect thinking" and "Psychological problems can occur at almost any age," which are about basic knowledge of mental health issues. One point is given for each correct answer, with incorrect or unknown responses receiving 0 points. The total scores range from 0 to 16 , with higher scores representing better health literacy. This scale can be used to monitor changes in mental health literacy over time as the mental health promotion activities envisaged. Previous studies have shown that this scale is a validated mental health literacy instrument (Liang et al., 2011). Wang et al. assessed the reliability and validity of the final version of this scale in Chinese adult community members and found that the internal consistency of the 16-item MHKQ was 0.59. In this study, Cronbach's alpha of the scale was $0.71, \mathrm{KOM}$ value of the scale was 0.71 .

\section{Social Support}

Social support was measured using the Social Support Rating Scale (SSRS) (Xiao ShuiYuan, 1987), designed by Xiao and colleagues. The SSRS is a self-report scale including ten items and three domains: Objective support, refering to an individual's social networks, and the instrumental and emotional support they have actually received in the past. Subjective support, refering to the emotional support experienced by the individual, that is, the emotional experience and satisfaction of being respected, supported and understood in the society. Social support utilization, refering to the extent to which support is utilized. Responses were provided using a 4-point Likert scale, with total scale scores ranging from 12 to 66, with higher scores indicating stronger social support. In the current 
study, the total score was divided into three levels: low (1222 ), moderate (23-44), and high social support (45-66). The SSRS has been shown to have good reliability and validity in Chinese patients with schizophrenia and is considered to be an easily understandable instrument to assess social support in Chinese populations (Tao et al., 2012). In the current study, Cronbach's alpha of the scale was $0.71, \mathrm{KOM}$ value of the scale was 0.70 .

\section{Loneliness}

Loneliness was measured by the University of California at Los Angeles loneliness scale (UCLA) (Russell, 1996). The scale included 20 items pertaining to loneliness, with 11 positive and 9 negative items, such as "How often do you feel that you lack companionship?" and "How often do you feel that you are "in tune" with the people around you?" to reflect how people sometimes feel. Each of the 20 items is rated on a scale of 1 (never), 2 (rarely), 3 (sometimes), and 4 (always). The range of possible scores was $20-80$, with higher scores signifying greater loneliness. The total score has been divided into three levels: mild loneliness (20-34), moderate loneliness (35-49), and severe loneliness (50-80). The Chinese version of the scale, translated by Wang et al., has demonstrated adequate reliability and validity in different populations including populations with schizophrenia (Wang, 1993; Schwartz and Gronemann, 2009). In this sample, Cronbach alpha was $0.79, \mathrm{KOM}$ value of the scale was 0.86 .

\section{Data Analytical Plan}

Data were entered using the Epidata3.1 database. Statistical analyses were conducted using SPSS version 20.0 (IBM, Chicago, IL, United States) for the descriptive analysis, linear regression analysis and Pearson correlation coefficient, and Mplus version 7.0 (Los Angeles, CA, United States) for the covariance structure analysis. The level of statistically significant differences was set at $p<0.05$.

For the first step, descriptive statistics (frequencies, percentages, means, and standard deviations) were calculated for socio-demographics and the key study variables, followed by Pearson correlations among these variables. Subsequently, a linear regression model was also used to analyze the influence of socio-demographics, in addition to health literacy and social support on loneliness. Ultimately, a structural equation model (SEM) was employed to further test the hypothesized relationships among health literacy, social support and loneliness of SMI patients (Baron and Kenny, 1986; Gunzler et al., 2013).

Using Baron and Kenny (1986) guidelines, to establish mediation, following steps need to be followed: (1) the independent variable (IV) predicts the presumed mediator variable (MV); (2) the MV predicts the dependent variable (DV), controlling for the IV; (3) after controlling for the effects of the MV, a previously significant relationship between the IV and the DV becomes non-significant (full or perfect mediation) or weaker (partial mediation). Thus, following the procedure proposed by Baron and Kenny, the analyses of the role of social support in mediating the relationship between health literacy and loneliness were planned in several stages (see Figure 2).

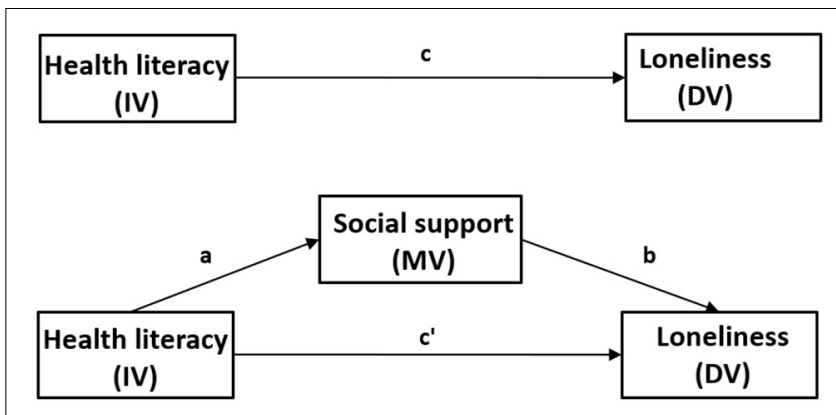

FIGURE 2 | Schematic model with social support as the mediator in the relationship between health literacy and loneliness. IV, independent variable (predictor); DV, dependent variable; MV, mediator variable. Path (c) represents the total effect of health literacy on loneliness (without considering the mediating effect of social support). Path (a) refers to the direct impact of health literacy on social support. Path (b) depicts the direct impact of social support on loneliness after controlling for health literacy. Path (c') represents the direct impact of health literacy on loneliness after controlling for social support. The indirect effect of health literacy on loneliness via social support is calculated as the product of the direct effects of health literacy on social support and social support on loneliness $(\mathrm{a} \times \mathrm{b})$.

\section{RESULTS}

\section{Sociodemographic Characteristics of the Participants}

Socio-demographic characteristics of the 270 SMI patients are displayed in Table 1. Of the total sample, the mean (SD) age of the total SMI patients was 49.7 (13.4) years and $44.1 \%$ were females. Almost a third were unmarried (31.1\%) and two third had elementary school education or less (62.2\%). About half of the SMI patients had a per capita annual household income of less than $\$ 750$ (48.5\%).

\section{Descriptive Analysis of Study Variables}

Table 2 shows the mean scores of the key variables for the entire sample. For health literacy, the mean score was $10.1 \pm 1.9$ and the computed score ranged from 4 to 16 . The proportion of respondents who answered each item correctly ranged from 26.3 to $97.4 \%$; the mean correct response rate for the 16 items was 63.1\%. As is shown in Table 2 and Figure 3, the mean score for social support was $30.2 \pm 7.2$. Based on the score, $13.0 \%$ (35), $83.7 \%$ (226), and $3.3 \%$ (9) of SMI patients had low, moderate, and high social support, respectively. As is shown in Table 3 and Figure 4, the mean score for loneliness was $45.8 \pm 8.0$, with $11.8 \%$ (32) of SMI patients experiencing mild loneliness, 53.0\% (143) and $35.2 \%$ (95) of SMI patients experiencing moderate and severe loneliness, respectively.

\section{Correlations of the Study Variable}

The Pearson's correlations for the study variables are presented in Table 3. Health literacy was positively correlated with social support and negatively correlated with loneliness. Social support was negatively correlated with loneliness. 
TABLE 1 | Socio-demographic characteristics of the patients with severe mental illness $(n=270)$.

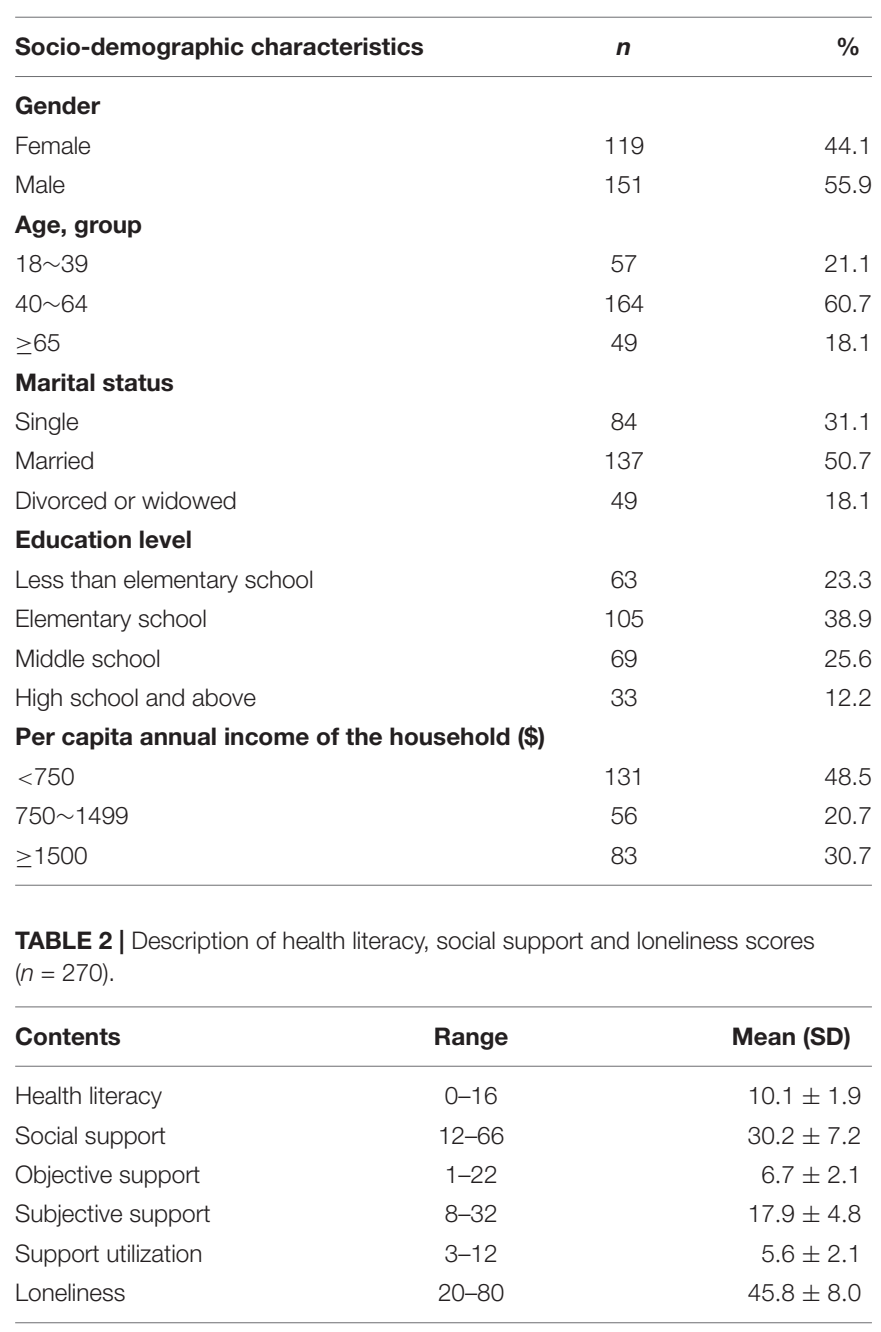

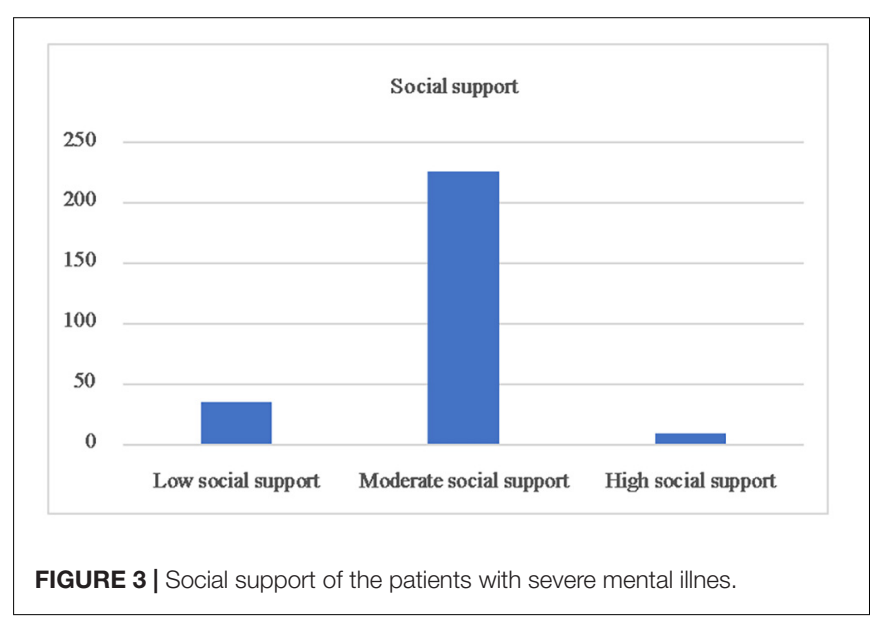

\section{Regression Analysis of Study Variable}

We used loneliness as the dependent variable and sociodemographic variables, health literacy and social support as independent variables in the linear regression model. Table 4
TABLE 3 | Correlation coefficients among study variables.

\begin{tabular}{lccc}
\hline Variables & (1) & (2) & (3) \\
\hline (1) Health literacy & & \\
(2) Social support & $0.328^{\star \star}$ & $-0.406^{\star \star}$ \\
(3) Loneliness & $-0.507^{\star \star}$ & \\
\hline
\end{tabular}

${ }^{* *} p<0.01$.

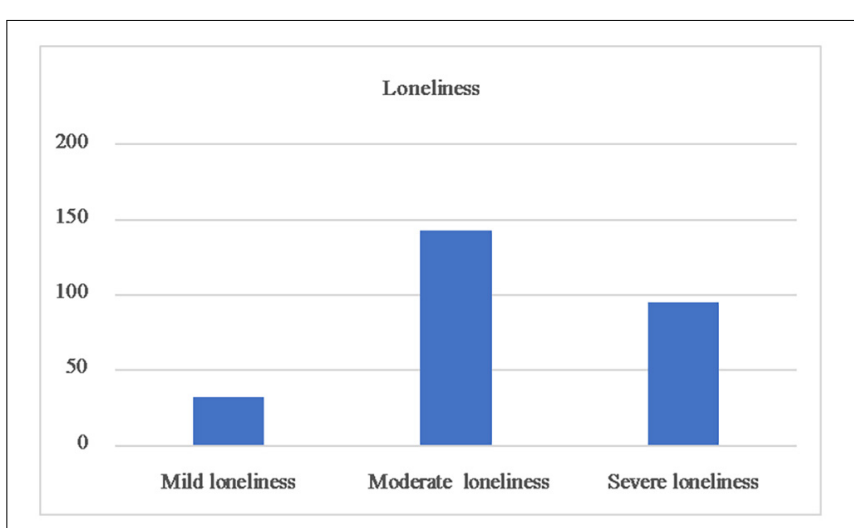

FIGURE 4 | Loneliness of the patients with severe mental illnes.

showed three socio-demographic factors in addition to health literacy and social support were significantly correlated with loneliness: gender, age, and marital status. Male SMI patients were more likely to endorse loneliness $(\beta=0.162, p=0.005)$. SMI patients aged 40 to $64(\beta=-0.137, p=0.044)$ were less likely to endorse loneliness when compared with those aged 18 to 39 . Married SMI patients $(\beta=-0.249, p<0.001)$ were less likely to be lonely when compared with single patients.

\section{Test of Study Model}

To examine the relationship between all of the variables, a structural equation model was estimated from the results of the linear regression analysis. Path analysis was further employed to test the hypothesized full model after controlling for the socio-demographics. With the addition of socio-demographics as covariates, the arrow direction among the core variables in the SEM remained unchanged and the corresponding coefficients did not change significantly. Thus, the socio-demographics were not confounding factors and the influence path of sociodemographics was not shown in the final model.

The SEM used bootstrap maximum likelihood estimation. The fit between the current data and the hypothesized model was assessed through several indicators: the comparative fit index (CFI) and the Tucker-Lewis index (TLI) of 0.97 or above; the root mean square error of approximation (RMSEA) and the standardized root mean square residual (SRMR) less than or equal to 0.05 and $\mathrm{a} \mathrm{x}^{2} / \mathrm{df}$ of $<3$, indicated an good model fit (Schermelleh-Engel et al., 2003). Figure 5 shows the final model where all paths were statistically significant and the model fit the data well: $\mathrm{CFI}=1.00$; TLI $=1.00$; $\mathrm{RMSEA}=0.00 ; \mathrm{SRMR}=0.00$ and $\mathrm{X}^{2} / \mathrm{df}<3.00$. 
TABLE 4 | Linear regression of factors associated with loneliness.

\begin{tabular}{|c|c|c|c|c|c|c|}
\hline \multirow[t]{2}{*}{ Factors } & \multicolumn{2}{|c|}{ Unstandardized coefficients } & \multirow{2}{*}{$\begin{array}{c}\text { Standardized coefficients } \\
\beta\end{array}$} & \multirow[t]{2}{*}{$t$} & \multirow[t]{2}{*}{$p$-value } & \multirow[t]{2}{*}{$95 \% \mathrm{Cl}$ for $\beta$} \\
\hline & $\beta$ & SE & & & & \\
\hline Constant & 75.880 & 2.929 & & 25.902 & $<0.001$ & $(70.111,81.649)$ \\
\hline \multicolumn{7}{|l|}{ Gender (ref: Female) } \\
\hline Male & 2.615 & 0.925 & 0.162 & 2.826 & 0.005 & $(0.792,4.437)$ \\
\hline \multicolumn{7}{|l|}{ Age (ref:18 39) } \\
\hline $40 \sim 64$ & -2.251 & 1.114 & -0.137 & -2.020 & 0.044 & $(-4.445,-0.057)$ \\
\hline$\geq 65$ & -2.409 & 1.448 & -0.116 & -1.664 & 0.097 & $(-5.260,0.442)$ \\
\hline \multicolumn{7}{|c|}{ Marital status (ref: Single) } \\
\hline Married & -4.319 & 1.154 & -0.249 & -3.743 & $<0.001$ & $(-6.591,-2.047)$ \\
\hline Divorced or widowed & -1.427 & 1.149 & -0.069 & -1.242 & 0.216 & $(-3.690,0.836)$ \\
\hline \multicolumn{7}{|c|}{ Education level (ref: Less than elementary school) } \\
\hline Elementary school & 0.592 & 1.071 & 0.036 & 0.552 & 0.581 & $(-1.518,2.701)$ \\
\hline Middle school & 1.143 & 1.204 & 0.062 & 0.950 & 0.343 & $(-1.227,3.514)$ \\
\hline High school and above & -1.746 & 1.518 & -0.071 & -1.151 & 0.251 & $(-4.735,1.243)$ \\
\hline \multicolumn{7}{|c|}{ Per capita annual income of household (ref: $<750, \$)$} \\
\hline $750 \sim 1,499$ & 1.969 & 1.053 & 0.099 & 1.870 & 0.063 & $(-0.105,4.044)$ \\
\hline$\geq 1,500$ & -0.757 & 0.951 & -0.044 & -0.796 & 0.427 & $(-2.630,1.116)$ \\
\hline Health literacy & -1.750 & 0.226 & -0.415 & -7.738 & $<0.001$ & $(-2.196,-1.305)$ \\
\hline Social support & -0.365 & 0.064 & -0.326 & -5.724 & $<0.001$ & $(-0.491,-0.239)$ \\
\hline
\end{tabular}

$R^{2}=0.36, F=13.77, p<0.001$.

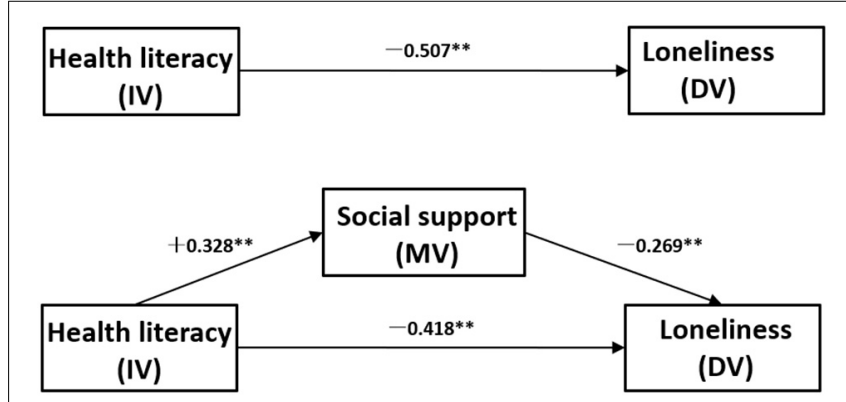

FIGURE 5 | The final model and standardized model paths.

Bootstrap with 2000 replications using maximum-likelihood estimation was employed for each path. The estimates for direct, indirect and total effects with bias-corrected 95\% CI are shown in Table 5. In these analyses, effect coefficients were substantially significant if the $95 \%$ CI does not include 0 (Hayes, 2009). The results showed that all path coefficients were substantially significant: Health literacy negativly predicts loneliness (c) $[\beta=-0.507,95 \% \mathrm{CI}:(-0.604)$ to $(-0.388)]$ and positively predicts social support (a) $(\beta=0.328,95 \% \mathrm{CI}$ : $0.201-0.451)$. Social support negativly predicts loneliness (b) $[\beta=-0.269,95 \% \mathrm{CI}:(-0.379)$ to $(-0.138)]$, controlling for health literacy. After controlling for the effects of social support, a previously significant relationship between health literacy and loneliness becomes weaker (c') $[\beta=-0.418,95 \%$ CI: $(-0.523)$ to $(-0.296)]$. Based on the above, health literacy negativly indirectly predicts loneliness through the partial mediating role of social support $(\mathrm{a} \times \mathrm{b})[\beta=-0.089,95 \% \mathrm{CI}:(-0.146)$ to $(-0.046)]$.
TABLE 5 | Direct, indirect and total effects and 95\% confidence intervals for the final model.

\begin{tabular}{lcc}
\hline Model pathways & Estimated effect & $95 \% \mathbf{C l}$ \\
\hline Total effects & & \\
Loneliness $<-$ Health literacy & -0.507 & $(-0.604)$ to $(-0.388)$ \\
Social support $<-$ Health literacy & 0.328 & 0.201 to 0.451 \\
Loneliness $<-$ Social support & -0.269 & $(-0.379)$ to $(-0.138)$ \\
Direct effects & & \\
Loneliness $<-$ Health literacy & -0.418 & $(-0.523)$ to $(-0.296)$ \\
Social support $<-$ Health literacy & 0.328 & $0.201-0.451$ \\
Loneliness $<-$ Social support & -0.269 & $(-0.379)$ to $(-0.138)$ \\
Indirect effects & & \\
Loneliness $<-$ Health literacy & -0.089 & $(-0.146)$ to $(-0.046)$
\end{tabular}

$\mathrm{Cl}$, confidence interval.

\section{DISCUSSION}

The current study examines the effect of health literacy and social support on loneliness among a sample of patients with SMI in China. In 270 SMI patients, $88.2 \%$ of SMI patients experienced moderate or more severe loneliness, which is significantly higher than Chinese older adults (19.6\%) (Yang, 2020) and adults with a diagnosis of psychosis in Australia (80\%) (Stain et al., 2012). People with SMI are often excluded socially because of their psychiatric symptoms (such as depression and paranoia) and the lack of social acceptance. In addition, patients themselves may feel inferior and actively isolate or create distance with other individuals by internalizing stigma (Prince et al., 2018). Thus, the experience of loneliness is particularly prevalent among patients with SMI, due to their deficiencies 
in certain physical, psychological, and social functioning skills (Schwartz and Gronemann, 2009).

The results of this research indicated that only $3.3 \%$ of the individuals with SMI identified a high level of social support, which was lower than previous surveys of the general population (56.9\%) (Jiangfei et al., 2017), indicating that the social support among this group is seriously impoverished. This finding is consist with previous research, which suggests that those with psychosis are more likely to have reduced social networks and limited access to satisfactory social support compared with others (Buchanan, 1995; Macdonald et al., 2000). In the Chinese context, social support is based on a strict social relationship network and is maintained through the reciprocate of favors (Kleinman and Kleinman, 1993). A diagnosis of SMI and its onsets were events of "losing face," which would isolate a family from mutual help so as to lose support (Yang and Kleinman, 2008). Low household income may also exacerbate caregiving burden and lead to poorer social support (Chiou et al., 2009). In current study, $69.2 \%$ of participants had a per capita annual income of household less than $\$ 1500$, lower than the per capita annual income of Chinese rural residents (\$2924) and urban residents (\$6127) in 2018 (Statictics, 2018). As expected, the model results confirmed the existence of a negative direct association between social support and loneliness among SMI patients which is consistent with the afore mentioned notion that social support acts as a protective factor by decreasing the feelings of loneliness (Institute of Medicine, 2004; Yildirim and Kocabiyik, 2010). The evidence presented by Sheridan and colleagues (Sheridan et al., 2015) and Zhang and colleagues (Zhang et al., 2018) indicate a clear trend that adequate social support and socializing interventions for people enduring mental illness lead to improved social functioning and reduced levels of loneliness. This suggests that to decrease loneliness in SMI patients, enhancing social support should be considered.

The mean overall health literacy (operationalized as the correct response rate on the MHKQ) of SMI patients in this study was $63.1 \%$, which is lower than that of the general public in China (77.0\%) (Wang et al., 2013). Cognitive or functional impairments which may accompany the presence of a mental illness, and poverty are established risk factors for low health literacy, which may help to explain this finding (Camann, 2001; Clausen et al., 2016). In addition, $62.2 \%$ of the participants in the sample had an elementary school education or less, representing a lower educational level than that of the overall general population in China (33.8\%), the rural population in China (45.3\%), and the rural population in Sichuan Province (54.7\%), as collected from The Sixth National Census (Statictics, 2010). The data indicates that the general education level among sample population in this study was low, which is typically associated with low health literacy (Yost et al., 2013). As hypothesized, the SEM results of the current study suggest that SMI patients with lower health literacy were more likely to endorse loneliness, consistent with the relationship reported by Geboers and colleagues (Geboers et al., 2016). Individuals with low health literacy not only lack knowledge around positive health behaviors or self-help strategies but also have difficulties recognizing their mental health issues and making successful health decisions (Pignone et al.,
2005; Kim et al., 2017; Degan et al., 2019b). Further, in China, where personal and social stigma toward mental illness is more prevalent than that in Western countries, individuals with low health literacy who experience SMI may be more likely to hide their plight due to shame and may withdraw from social contacts for fear of rejection (Parikh et al., 1996; Corrigan, 2015; Zhuang et al., 2017). These findings highlight the need to increase overall health literacy of SMI patients and confirm the importance of health literacy in decreasing feelings of loneliness.

The model results suggest that SMI patients' health literacy is direct positively associated with their social support: the higher the levels of health literacy, the better the levels of social support, which has been supported by previous studies (Lee et al., 2018; Degan et al., 2019a). Improving health literacy can be considered as a possible public health strategy for encouraging help-seeking, those who have better health literacy may be more likely to acquire adequate support and apply their resources to solving their health problems (Suka et al., 2015). The most significant finding of this study was the partial mediating role of social support in the relationship between health literacy and loneliness. Our results suggest that low health literacy is directly related to SMI patients' loneliness; and it can indirectly affect loneliness through the mediation of social support. Furthermore, effect contrasts indicated that the direct effect of health literacy was statistically significantly greater than that of social support. Thus, to decrease the loneliness level of SMI patients, health literacy can be regarded as an important intervening target.

According to Brijnath et al. (2016), psychoeducation and abnormal cognitive-behavioral therapy for SMI patients may be beneficial toward improving health literacy and ultimately reducing self perceived lonliness. Meanwhile, education and therapy strategies should take into account the understanding and receptivity of SMI patients with low education level. Other possible approaches are to increase patients' opportunities for social contact, improve their social networks and enhance social support received from family and friends (Lee et al., 2004). However, if members of an individuals social network have inadequate knowledge about mental illness, their support could be unhelpful or even harmful (Griffiths et al., 2011). Therefore, public education campaigns for patients' proxies or direct caregivers are also needed.

Of note, results of the present study indicated that the gender, age and marital status of SMI patients were associated with loneliness. Males with SMI were more likely to endorse loneliness, which is in line with the previous research (van den Broek, 2017). Men may have less multifaceted social networks resulting in poorer social support (Zhou et al., 2018). In addition, SMI patients who were middle-aged or patients who were married in this study were less likely to be lonely, which is consistent with previous research (Perlman and Peplau, 1984; Luhmann and Hawkley, 2016). One possible explanation may be that being unmarried, not having a partner or living alone, means that needs for emotional or instrumental support are more likely to be unmet, thus increasing the risk of loneliness. Another possible explanation may be that young adults may have higher expectations about their social networks and interactions, while middle-aged adults have more accumulated resources with age, 
may be more easily satisfied with their social relations or there is greater alignement between their acutal and expected social interactions (Perlman and Peplau, 1984). This suggests that more attention should be paid to male, young and single individuals with SMI with regard to social support and lonliness. The study did not find the relationship between loneliness and educational level of SMI patients, which is consistent with previous research (Ferreira-Alves et al., 2014). Contrary to previous studies (Nicolaisen and Thorsen, 2014), the linear regression results of this study showed that there is no association between SMI patients' per capita annual income of household and lonliness. Future Studies can further explore the specific impact of income and its related factors on loneliness in SMI patients.

The examination of factors which contribute to loneliness among people with SMI is important. Addressing loneliness might improve general health, and can lead to positive patient centered outcomes (e.g., reduction in behavioral health symptoms, recovery) and reduce negative health outcomes (e.g., re-hospitalization). A limited number of studies have discussed the relationship between health literacy and loneliness and no study, to our knowledge, has addressed these variables in SMI patients. The present study emphasized the role of health literacy and social support as the predictors of loneliness among individuals with SMI. Findings of this study enrich the explanation of the mechanism supporting the association between health literacy and loneliness and can be referenced to ameliorating the loneliness of the individuals with SMI. The main limitations of this study also need to be considered. First, the participants in the present study are representative of individuals with SMI in Southwest China and findings might differ in other populations, urban areas or other countries. Second, the questionnaire in this study was conducted by self-report, and the respondents may have some report bias. Third, most of the scales appear to have low internal consistency scores, low internal consistency of the measures makes it difficult for us to assume that the variance explained is actually due to the measures themselves. And as we did not collect data relating to mental health diagnosis and medication information, it is not clear if our findings might be impacted by these background information of SMI patients. Furthermore, the cross-sectional nature of the data makes it impossible to allow conclusions on causality. Future Longitudinal or experiment studies may be conducted provide more definite information about the causal inference.

\section{CONCLUSION}

In conclusion, this study aimed to investigate the relationships among health literacy, social support, and loneliness among Chinese individuals with SMI. Results suggest that health litercay and social support exert a significant direct negative influence on loneliness. The results also suggest that social support played

\section{REFERENCES}

Barkus, E., and Badcock, J. C. (2019). A transdiagnostic perspective on social anhedonia. Front. Psychiatry 10:216. doi: 10.3389/fpsyt.2019.00216 a mediating role in the association between health literacy and loneliness. It is likely that the influence of low health literacy on loneliness could be buffered further by adequate social support, highlighting the importance of the identification of interventions targeting health literacy and social support which could ultimately decrease perceptions of lonliness among SMI patients. Psychoeducation and abnormal cognitive-behavioral therapy for SMI patients, education campaigns for patients' proxies or direct caregivers may be beneficial to this end. For the socio-demographic characteristics, male,youth and single were predictive of higher levels of SMI patients' loneliness. Future interventions may also target males, young adults and single SMI patients as they were found in the current study to have higher levels of lonliness.

\section{DATA AVAILABILITY STATEMENT}

The original contributions presented in the study are included in the article/Supplementary Material, further inquiries can be directed to the corresponding author/s.

\section{ETHICS STATEMENT}

The studies involving human participants were reviewed and approved by Institutional Review Board of Sichuan University. The patients/participants provided their written informed consent to participate in this study.

\section{AUTHOR CONTRIBUTIONS}

DL conceived the study and prepared the study protocol. YL performed the data analysis and drafted the first manuscript. All authors contributed to content revisions, approved the final version, and agreed to be responsible for all aspects of the current work.

\section{ACKNOWLEDGMENTS}

We first of all wish to thank the respondents for participating in the study. We are also grateful to all the investigators for their assistance with data collection.

\section{SUPPLEMENTARY MATERIAL}

The Supplementary Material for this article can be found online at: https://www.frontiersin.org/articles/10.3389/fpsyg. 2021.564666/full\#supplementary-material

Baron, R. M., and Kenny, D. A. (1986). The moderator-mediator variable distinction in social psychological research: conceptual, strategic, and statistical considerations. J. Pers. Soc. Psychol. 51, 1173-1182. doi: 10.1037//0022-3514.51. 6.1173 
Brijnath, B., Protheroe, J., Mahtani, K. R., and Antoniades, J. (2016). Do web-based mental health literacy interventions improve the mental health literacy of adult consumers? Results from a systematic review. J. Med. Internet Res. 18:e165. doi: 10.2196/jmir.5463

Buchanan, J. (1995). Social support and schizophrenia: a review of the literature. Arch. Psychiatr. Nurs. 9, 68-76. doi: 10.1016/s0883-9417(95)80003-4

Cacioppo, J. T., Fowler, J. H., and Christakis, N. A. (2009). Alone in the crowd: the structure and spread of loneliness in a large social network. J. Pers. Soc. Psychol. 97, 977-991. doi: 10.1037/a0016076

Camann, M. A. (2001). To your health: implementation of a wellness program for treatment staff and persons with mental illness. Arch. Psychiatr. Nurs. 15, 182-187. doi: 10.1053/apnu.2001.25418

Chiou, C. J., Chang, H. Y., Chen, I. P., and Wang, H. H. (2009). Social support and caregiving circumstances as predictors of caregiver burden in Taiwan. Arch. Gerontol. Geriatr. 48, 419-424. doi: 10.1016/j.archger.2008.04.001

Chrostek, A., Grygiel, P., Anczewska, M., Wciorka, J., and Switaj, P. (2016). The intensity and correlates of the feelings of loneliness in people with psychosis. Compr. Psychiatry 70, 190-199. doi: 10.1016/j.comppsych.2016.07.015

Clausen, W., Watanabe-Galloway, S., Bill Baerentzen, M., and Britigan, D. H. (2016). Health literacy among people with serious mental illness. Community Ment. Health J. 52, 399-405. doi: 10.1007/s10597-015-9951-8

Corrigan, P. W. (2015). On the stigma of mental illness: practical strategies for research and social change. Child Family Behav. Ther. 57, 1048-1048.

Degan, T. J., Kelly, P. J., Robinson, L. D., and Deane, F. P. (2019a). Health literacy in substance use disorder treatment: a latent profile analysis. J. Subst. Abuse Treat. 96, 46-52. doi: 10.1016/j.jsat.2018.10.009

Degan, T. J., Kelly, P. J., Robinson, L. D., Deane, F. P., Wolstencroft, K., Turut, S., et al. (2019b). Health literacy in people living with mental illness: a latent profile analysis. Psychiatry Res. 280:112499. doi: 10.1016/j.psychres.2019.112499

Ferreira-Alves, J., Magalhães, P., Viola, L., and Simoes, R. (2014). Loneliness in middle and old age: demographics, perceived health, and social satisfaction as predictors. Arch. Gerontol. Geriatr. 59, 613-623. doi: 10.1016/j.archger.2014.06. 010

Gayer-Anderson, C., and Morgan, C. (2013). Social networks, support and early psychosis: a systematic review. Epidemiol. Psychiatr. Sci. 22, 131-146. doi: 10. 1017/s2045796012000406

Geboers, B., Reijneveld, S. A., Jansen, C. J., and de Winter, A. F. (2016). Health literacy is associated with health behaviors and social factors among older adults: results from the lifelines cohort study. J. Health Commun. 21, 45-53. doi: 10.1080/10810730.2016.1201174

Griffiths, K. M., Crisp, D. A., Barney, L., and Reid, R. (2011). Seeking help for depression from family and friends: a qualitative analysis of perceived advantages and disadvantages. BMC Psychiatry 11:196. doi: 10.1186/1471-244x11-196

Gunzler, D., Chen, T., Wu, P., and Zhang, H. (2013). Introduction to mediation analysis with structural equation modeling. Shanghai Arch. Psychiatry 25, 390394. doi: 10.3969/j.issn.1002-0829.2013.06.009

Hawkley, L. C., and Cacioppo, J. T. (2010). Loneliness matters: a theoretical and empirical review of consequences and mechanisms. Ann. Behav. Med. 40, 218-227. doi: 10.1007/s12160-010-9210-8

Hayes, A. F. (2009). Beyond Baron and Kenny: statistical mediation analysis in the new millennium. Commun. Monogr. 76, 408-420.

Hyman, S. E., Andrews, G., Ayuso-Mateos, J. L., Gaebel, W., and Reed, G. (2011). A conceptual framework for the revision of the ICD-10 classification of mental and behavioural disorders. World Psychiatry 10, 86-92.

Institute of Medicine (2004). "Committee on health literacy board on neuroscience and behavioral health," in Health Literacy: A Prescription to End Confusion, eds L. Nielsen-Bohlman, A. M. Panzer, and D. A. Kindig (Washington,DC: National Academies Press).

Jansen, M. A. (2018). Psychosocial services for individuals with serious mental illness/severe emotional disturbance: clinical practice guideline toolkit. Psychiatry 81, 3-21. doi: 10.1080/00332747.2018.1440116

Jiangfei, L., Xiangxiang, Z., Shan, Z., Zhiqiang, Z., Lei, J., and Yue, L. (2017). Correlation of social support and mental health among residents in Weifang. Chinese Med. Ethics 30:4.

Kang, H. W., Park, M., and Wallace Hernandez, J. P. (2018). The impact of perceived social support, loneliness, and physical activity on quality of life in
South Korean older adults. J. Sport Health Sci. 7, 237-244. doi: 10.1016/j.jshs. 2016.05.003

Kim, Y. S., Lee, H. Y., Lee, M. H., Simms, T., and Park, B. H. (2017). Mental health literacy in korean older adults: a cross-sectional survey. J. Psychiatr. Ment. Health Nurs. 24, 523-533. doi: 10.1111/jpm.12395

Kleinman, A., and Kleinman, J. (1993). Face, favor and families: the social course of mental health problems in Chinese and American societies. Chinese J. Ment. Health 6, 37-47.

Lee, J. Y., Murry, N., Ko, J., and Kim, M. T. (2018). Exploring the Relationship between maternal health literacy, parenting self-efficacy, and early parenting practices among low-income mothers with infants. J. Health Care Poor Underserved 29, 1455-1471. doi: 10.1353/hpu.2018.0106

Lee, S. Y., Arozullah, A. M., and Cho, Y. I. (2004). Health literacy, social support, and health: a research agenda. Soc. Sci. Med. 58, 1309-1321. doi: 10.1016/s02779536(03)00329-0

Liang, X., Zhu, X., and Liu, T. (2011). Cross-sectional survey on awareness of mental health knowledge among communities in Xi'an. China J. Health Psychol. 25, 1200-1202.

Linz, S. J., and Sturm, B. A. (2013). The phenomenon of social isolation in the severely mentally ill. Perspect. Psychiatr. Care 49, 243-254. doi: 10.1111/ppc. 12010

Lora, C. M., Gordon, E. J., Sharp, L. K., Fischer, M. J., Gerber, B. S., and Lash, J. P. (2011). Progression of CKD in Hispanics: potential roles of health literacy, acculturation, and social support. Am. J. Kidney Dis. 58, 282-290. doi: 10.1053/ j.ajkd.2011.05.004

Luhmann, M., and Hawkley, L. C. (2016). Age differences in loneliness from late adolescence to oldest old age. Dev. Psychol. 52, 943-959. doi: 10.1037/ dev0000117

Macdonald, E. M., Hayes, R. L., and Baglioni, A. J. Jr. (2000). The quantity and quality of the social networks of young people with early psychosis compared with closely matched controls. Schizophr. Res. 46, 25-30. doi: 10.1016/s09209964(00)00024-4

Masi, C. M., Chen, H. Y., Hawkley, L. C., and Cacioppo, J. T. (2011). A metaanalysis of interventions to reduce loneliness. Pers. Soc. Psychol. Rev. 15, 219-266. doi: 10.1177/1088868310377394

McAuley, E., Blissmer, B., Marquez, D. X., Jerome, G. J., Kramer, A. F., and Katula, J. (2000). Social relations, physical activity, and well-being in older adults. Prev. Med. 31, 608-617. doi: 10.1006/pmed.2000.0740

Medicine (2004). Health literacy: a prescription to end confusion. 15, 389-395.

Meltzer, H., Bebbington, P., Dennis, M. S., Jenkins, R., McManus, S., and Brugha, T. S. (2013). Feelings of loneliness among adults with mental disorder. Soc. Psychiatry Psychiatr. Epidemiol. 48, 5-13. doi: 10.1007/s00127-012-0515-8

Nguyen, T., Tran, T., Green, S., Hsueh, A., Tran, T., Tran, H., et al. (2020). Proof of concept of participant informed, psycho-educational, communitybased intervention for people with severe mental illness in rural Vietnam. Int. J. Soc. Psychiatry 66, 232-239. doi: 10.1177/0020764019898234

Nicolaisen, M., and Thorsen, K. (2014). Loneliness among men and women-a fiveyear follow-up study. Aging Ment. Health 18, 194-206. doi: 10.1080/13607863. 2013.821457

Pan, J., Ren, Z., Li, W., Wei, Z., Rao, H., Ren, H., et al. (2018). Prevalence of hyperlipidemia in Shanxi Province, China and application of Bayesian networks to analyse its related factors. Sci. Rep. 8:3750. doi: 10.1038/s41598-018-22167-2

Parikh, N. S., Parker, R. M., Nurss, J. R., Baker, D. W., and Williams, M. V. (1996). Shame and health literacy: the unspoken connection. Patient Educ. Couns. 27, 33-39. doi: 10.1016/0738-3991(95)00787-3

Perese, E. F., and Wolf, M. (2005). Combating loneliness among persons with severe mental illness: social network interventions' characteristics, effectiveness, and applicability. Issues Ment. Health Nurs. 26, 591-609. doi: 10.1080/ 01612840590959425

Perlman, D., and Peplau, L. (1984). "Loneliness research: a survey of empirical findings," in Preventing the Harmful Consequences of Severe and Persistent Loneliness, eds L. A. Peplau and S. E. Goldston (Washington, D.C: US Goverment PrIntIng OffIce), 13-46.

Pignone, M., DeWalt, D. A., Sheridan, S., Berkman, N., and Lohr, K. N. (2005). Interventions to improve health outcomes for patients with low literacy. A systematic review. J. Gen. Intern. Med. 20, 185-192. doi: 10.1111/j.1525-1497. 2005.40208.x 
Prince, J. D., Oyo, A., Mora, O., Wyka, K., and Schonebaum, A. D. (2018). Loneliness among persons with severe mental illness. J. Nerv. Ment. Dis. 206, 136-141. doi: 10.1097/nmd.0000000000000768

Roe, D., Mashiach-Eizenberg, M., and Lysaker, P. H. (2011). The relation between objective and subjective domains of recovery among persons with schizophrenia-related disorders. Schizophr. Res. 131, 133-138. doi: 10.1016/j. schres.2011.05.023

Russell, D. W. (1996). UCLA loneliness scale (Version 3): reliability, validity, and factor structure. J. Pers. Assess. 66, 20-40. doi: 10.1207/s15327752jpa6601_2

Schermelleh-Engel, K., Moosbrugger, H., and Müller, H. (2003). Evaluating the fit of structural equation models: tests of significance and descriptive goodness-offit measures. Methods Psychol. Res. 8, 23-74.

Schwartz, C., and Gronemann, O. C. (2009). The contribution of self-efficacy, social support and participation in the community to predicting loneliness among persons with schizophrenia living in supported residences. Isr. J. Psychiatry Relat. Sci. 46, 120-129.

Sheridan, A. J., Drennan, J., Coughlan, B., O’Keeffe, D., Frazer, K., Kemple, M., et al. (2015). Improving social functioning and reducing social isolation and loneliness among people with enduring mental illness: report of a randomised controlled trial of supported socialisation. Int. J. Soc. Psychiatry 61, 241-250. doi: 10.1177/0020764014540150

Stain, H. J., Galletly, C. A., Clark, S., Wilson, J., Killen, E. A., Anthes, L., et al. (2012). Understanding the social costs of psychosis: the experience of adults affected by psychosis identified within the second Australian national survey of psychosis. Aust. N. Z. J. Psychiatry 46, 879-889. doi: 10.1177/0004867412449060

Statictics (2010). The China's 2010 Census-Volume 3: Education Level [Online]. Beijing: Statistics Press.

Statictics (2018). China Statistical Yearbook 2018. Beijing: China Statistics Press.

Stewart, D. W., Reitzel, L. R., Correa-Fernandez, V., Cano, M. A., Adams, C. E., Cao, Y., et al. (2014). Social support mediates the association of health literacy and depression among racially/ethnically diverse smokers with low socioeconomic status. J. Behav. Med. 37, 1169-1179. doi: 10.1007/s10865-0149566-5

Suka, M., Yamauchi, T., and Sugimori, H. (2015). Relationship between individual characteristics, neighbourhood contexts and help-seeking intentions for mental illness. BMJ Open 5:e008261. doi: 10.1136/bmjopen-2015-008261

Tao, H., Song, L., Niu, X., Li, X., Zhang, Q., Cui, J., et al. (2012). Effectiveness of a rehabilitative program that integrates hospital and community services for patients with schizophrenia in one community in Shanghai. Shanghai Arch. Psychiatry 24, 140-148. doi: 10.3969/j.issn.1002-0829.2012.03.003

Tharayil, D. P. (2007). Self, social, and family perceptions in relation to loneliness controlling for depression among individuals with Schizophrenia. Internet $J$. Ment. Health 4, 1-21.

van den Broek, T. (2017). Gender differences in the correlates of loneliness among Japanese persons aged 50-70. Austr. J. Ageing 36, 234-237. doi: 10.1111/ajag. 12448

van der Werf, M., van Winkel, R., van Boxtel, M., and van Os, J. (2010). Evidence that the impact of hearing impairment on psychosis risk is moderated by the level of complexity of the social environment. Schizophr. Res. 122, 193-198. doi: 10.1016/j.schres.2010.05.020

Vigo, D., Thornicroft, G., and Atun, R. (2016). Estimating the true global burden of mental illness. Lancet Psychiatry 3, 171-178. doi: 10.1016/s2215-0366(15) 00505-2

Wang, D., Ma, J., Tan, L., Chen, Y., Li, X., Tian, X., et al. (2017). Epidemiology of severe mental illness in Hunan province in central China during 2014-2015: a multistage cross-sectional study. PLoS One 12:e0188312. doi: 10.1371/journal. pone. 0188312

Wang, J., He, Y., Jiang, Q., Cai, J., Wang, W., Zeng, Q., et al. (2013). Mental health literacy among residents in Shanghai. Shanghai Arch. Psychiatry 25, 224-235. doi: 10.3969/j.issn.1002-0829.2013.04.004
Wang, X. D. (1993). Handbook of mental health assessment scale. Chinese Ment. Health J. 5, 251-259.

Wong, M. M., Castle, D., Organ, B., Li, J., Ma, L., Chui, E., et al. (2020). Present and future of severe mental illness (SMI) policies: reflections from an Asia-Pacific expert forum series. Psychiatry Clin. Neurosci. 74:156. doi: 10.1111/pcn.12964

Xiao ShuiYuan, Y. D. (1987). The effect of social support on physical and psychological health. J. Chin. Psychiatry 4, 183-187.

Yang, F. (2020). Widowhood and loneliness among Chinese older adults: the role of education and gender. Aging Ment. Health 2020, 1-10. doi: 10.1080/13607863. 2020.1732293

Yang, L. H., and Kleinman, A. (2008). 'Face' and the embodiment of stigma in China: the cases of schizophrenia and AIDS. Soc. Sci. Med. 67, 398-408. doi: 10.1016/j.socscimed.2008.03.011

Yang, Y., Zhang, B., Meng, H., Liu, D., and Sun, M. (2019). Mediating effect of social support on the associations between health literacy, productive aging, and self-rated health among elderly Chinese adults in a newly urbanized community. Medicine 98, e15162. doi: 10.1097/md.00000000000 15162

Yildirim, Y., and Kocabiyik, S. (2010). The relationship between social support and loneliness in Turkish patients with cancer. J. Clin. Nurs. 19, 832-839. doi: 10.1111/j.1365-2702.2009.03066.x

Yost, K. J., DeWalt, D. A., Lindquist, L. A., and Hahn, E. A. (2013). The association between health literacy and indicators of cognitive impairment in a diverse sample of primary care patients. Patient Educ. Couns. 93, 319-326. doi: 10.1016/ j.pec.2013.07.006

Yu, Y., Liu, Z. W., Tang, B. W., Zhao, M., Liu, X. G., and Xiao, S. Y. (2017). Reported family burden of schizophrenia patients in rural China. PLoS One 12:e179425. doi: 10.1371/journal.pone.0179425

Zhang, S., Tian, Y., Sui, Y., Zhang, D., Shi, J., Wang, P., et al. (2018). Relationships between social support, loneliness, and internet addiction in chinese postsecondary students: a longitudinal cross-lagged analysis. Front. Psychol. 9:1707. doi: 10.3389/fpsyg.2018.01707

Zhou, Z., Wang, P., and Fang, Y. (2018). Loneliness and the risk of dementia among older Chinese adults: gender differences. Aging Ment. Health 22, 519-525. doi: 10.1080/13607863.2016.1277976

Zhuang, X. Y., Wong, D. F. K., Cheng, C. W., and Pan, S. M. (2017). Mental health literacy, stigma and perception of causation of mental illness among Chinese people in Taiwan. Int. J. Soc. Psychiatry 63, 498-507. doi: 10.1177/ 0020764017719303

Zou, J. (2014). Associations between trait emotional intelligence and loneliness in Chinese undergraduate students: mediating effects of self-esteem and social support. Psychol. Rep. 114, 880-890. doi: 10.2466/04.21.PR0.11 $4 \mathrm{k} 29 \mathrm{w} 3$

Conflict of Interest: The authors declare that the research was conducted in the absence of any commercial or financial relationships that could be construed as a potential conflict of interest.

Publisher's Note: All claims expressed in this article are solely those of the authors and do not necessarily represent those of their affiliated organizations, or those of the publisher, the editors and the reviewers. Any product that may be evaluated in this article, or claim that may be made by its manufacturer, is not guaranteed or endorsed by the publisher.

Copyright (c) 2021 Liu, Meng, Conner, Qiao and Liu. This is an open-access article distributed under the terms of the Creative Commons Attribution License (CC BY). The use, distribution or reproduction in other forums is permitted, provided the original author(s) and the copyright owner(s) are credited and that the original publication in this journal is cited, in accordance with accepted academic practice. No use, distribution or reproduction is permitted which does not comply with these terms. 